\title{
Editorial
}

\section{Functional Differential and Difference Equations with Applications 2013}

\author{
J. Diblík, ${ }^{1}$ E. Braverman, ${ }^{2}$ I. Györi, ${ }^{3}$ Yu. Rogovchenko, ${ }^{4}$ M. Růžičková, ${ }^{5}$ and A. Zafer ${ }^{6}$ \\ ${ }^{1}$ Brno University of Technology, Brno, Czech Republic \\ ${ }^{2}$ University of Calgary, Calgary, Canada \\ ${ }^{3}$ University of Pannonia, Veszprém, Hungary \\ ${ }^{4}$ University of Agder, Kristiansand, Norway \\ ${ }^{5}$ University of Žilina, Žilina, Slovakia \\ ${ }^{6}$ Middle East Technical University, Ankara, Turkey \\ Correspondence should be addressed to J. Diblík; diblik@feec.vutbr.cz
}

Received 27 January 2014; Accepted 27 January 2014; Published 12 May 2014

Copyright (C) 2014 J. Diblík et al. This is an open access article distributed under the Creative Commons Attribution License, which permits unrestricted use, distribution, and reproduction in any medium, provided the original work is properly cited.

This annual issue comes as a sequel to two special issues, Recent Progress in Differential and Difference Equations edited by the four members of the present team and Functional Differential and Difference Equations with Applications with the same editorial team, both published by the Abstract and Applied Analysis in 2011 and 2012, respectively.

In the call for papers prepared by the Guest Editors and posted on the journal's web page, we encouraged submission of state-of-the-art contributions on a wide spectrum of topics such as asymptotic behavior of solutions, boundedness and periodicity of solutions, nonoscillation and oscillation of solutions, representation of solutions, stability, numerical algorithms, and computational aspects, as well as applications to real-world phenomena. This invitation was warmly welcomed by the mathematical community; more than sixty manuscripts addressing important problems in the abovementioned fields of qualitative theory of functional differential and difference equations were submitted to the Editorial Office and went through a thorough peer refereeing process. Twenty-seven carefully selected research articles collected in this special issue reflect modern trends and advances in functional differential and difference equations. Sixty-seven authors from fourteen countries (China, Czech Republic, Georgia, Hungary, Israel, Latvia, Norway, Portugal, Saudi Arabia, Serbia, Slovak Republic, Spain, Turkey, and Ukraine) have contributed to the success of this thematic collection of papers.
Questions related to the existence of periodic solutions and their stability properties traditionally attract attention of researchers working in the qualitative theory of differential, functional differential, and difference equations. In this issue, the reader will find results that relate periodicity of linear autonomous nonhomogeneous difference equations to the existence of equilibria. Systems of nonlinear difference equations whose all well-defined solutions are periodic are considered. For some classes of nonlinear systems with delay, it is shown that the presence of the time delay results in the existence of periodic solutions.

Existence of oscillating solutions for half-linear differential equations with delay and for even order damped equations with distributed deviating arguments is studied. Nonlinear oscillations in the context of saddle-center bifurcation in the dynamical system describing a singularly perturbed forced oscillator of Duffing's type with a nonlinear restoring and a nonperiodic external driving force are examined. Boundedness of solutions to a class of second-order periodic systems with singularities is considered as well.

Stability problems always attract interest of researchers, and this special issue is not an exception. The reader will find papers on the stability of impulsive stochastic functional differential equations with delayed impulses and stability of differential systems under permanently arising impulses, an application of moment equations in a model of the stable foreign currency exchange market in conditions of 
uncertainty, a study on the global exponential stability of equilibria for impulsive cellular neural network models with piecewise alternately advanced and retarded arguments, and analysis of stability and global Hopf bifurcation phenomena in a ratio-dependent predator-prey model with two time delays.

New results for boundary value problems are also reported, including the existence and uniqueness theorem for solutions of nonhomogeneous impulsive boundary value problem for planar Hamiltonian systems and general results on the solvability of singular initial value problems.

Several articles deal with the behavior of solutions at infinity. Namely, explicit formulas for planar weakly delayed linear discrete systems are derived; results on asymptotic behavior of solutions to generalized Emden-Fowler differential equations with delayed argument, higher-order quasilinear neutral differential equations, vector integral equations with deviating arguments, and linear Volterra integrodifferential systems are reported along with the applications of nonlinear weakly singular inequalities to Volterra-type difference equations.

Other important problems discussed in this special issue are related to the representation of the solutions of linear discrete systems with constant coefficients and two delays, automorphisms of ordinary differential equations, spatial discretization of the Cauchy problem for a multidimensional linear parabolic partial differential equation of the second order with nondivergent operator, and unbounded timeand space-dependent coefficients. Newton-Kantorovich and Smale type convergence theorems are used in a deformed Newton method with the third-order convergence for solving nonlinear equations. Regularity of a mild solution for a stochastic fractional delayed reaction-diffusion equation driven by Lévy space-time white noise and an inverse problem for Dirac differential operators with discontinuity conditions and discontinuous coefficient are studied. Finally, a mathematical model for the incompressible twodimensional/axisymmetric non-Newtonian fluid flows and heat transfer analysis in the region of stagnation point over a stretching/shrinking sheet and axisymmetric shrinking sheet is presented.

It is not possible to provide in this short editorial note a detailed description for all papers included in this volume. However, it is clear that they reflect contemporary trends in the development of the qualitative theory of functional differential equations and feature important applications. We believe that this special issue challenges researchers with new unsolved problems and introduces many new ideas and useful techniques.

J. Diblik

E. Braverman

I. Györi

Yu. Rogovchenko

M. Rưžičková

A. Zafer 


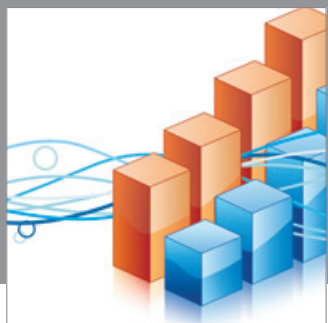

Advances in

Operations Research

mansans

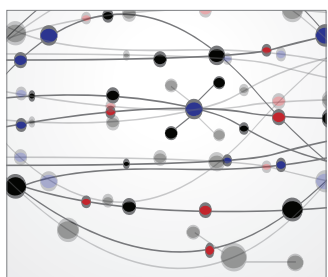

The Scientific World Journal
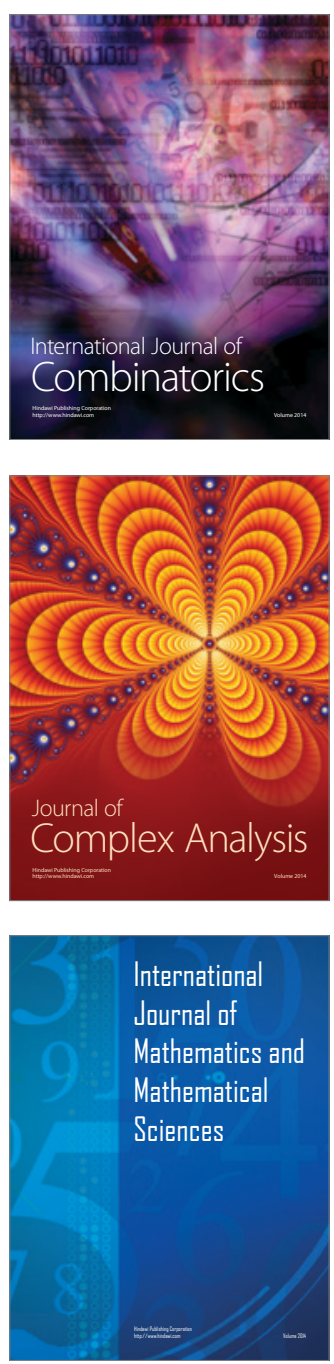
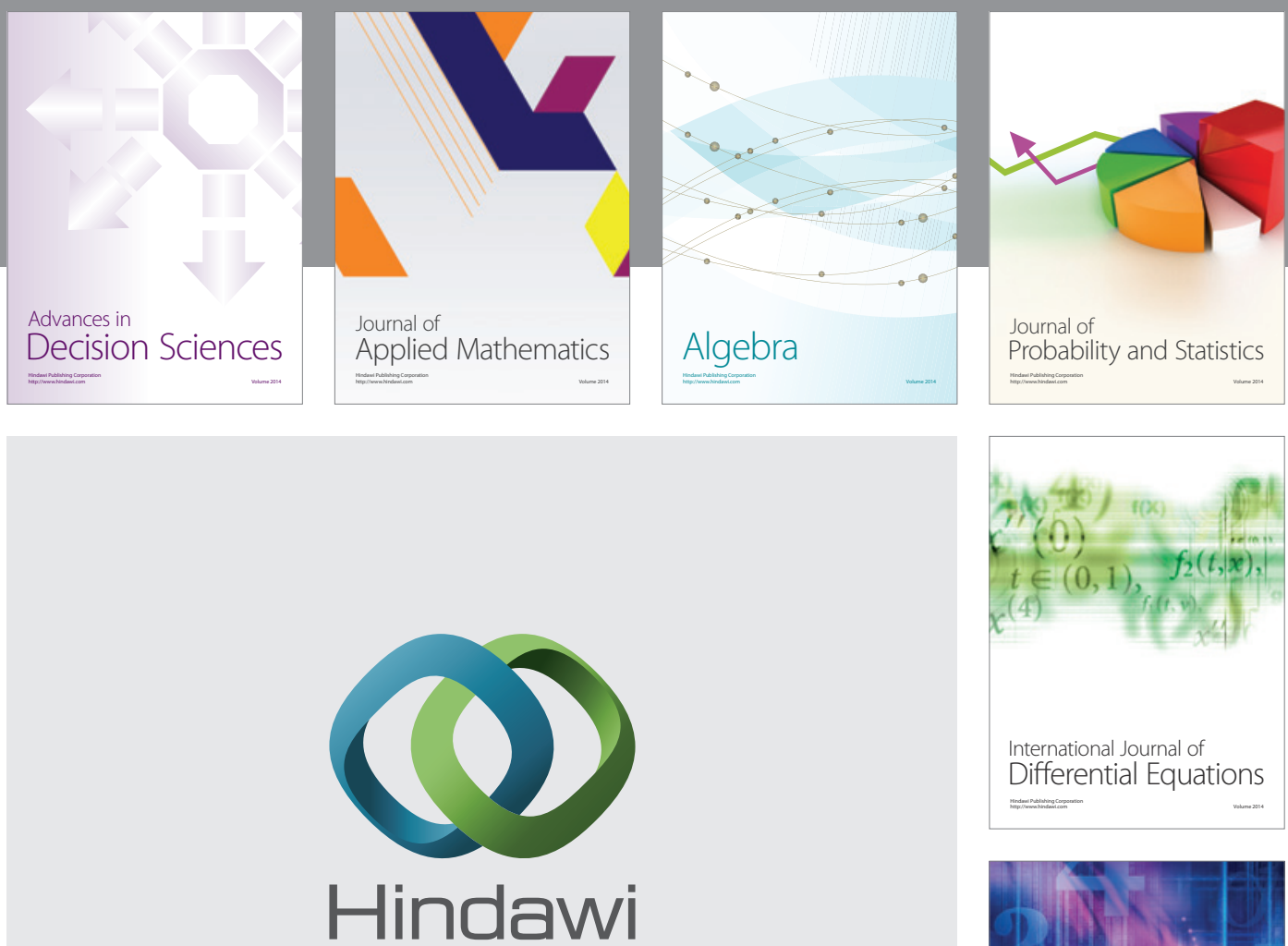

Submit your manuscripts at http://www.hindawi.com
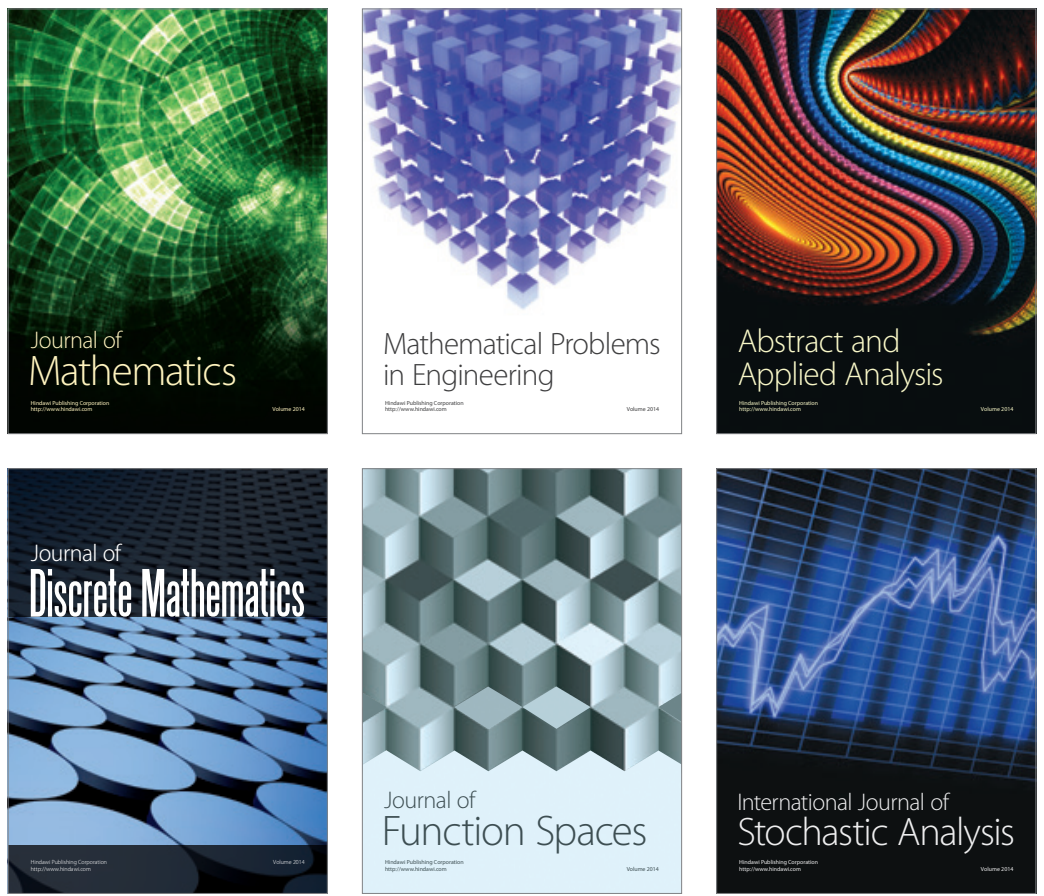

Journal of

Function Spaces

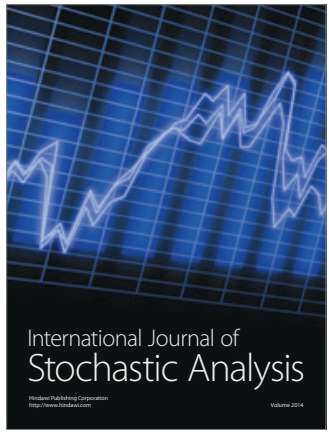

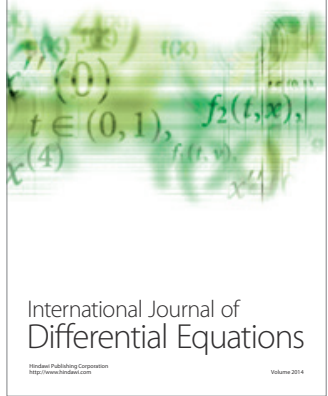
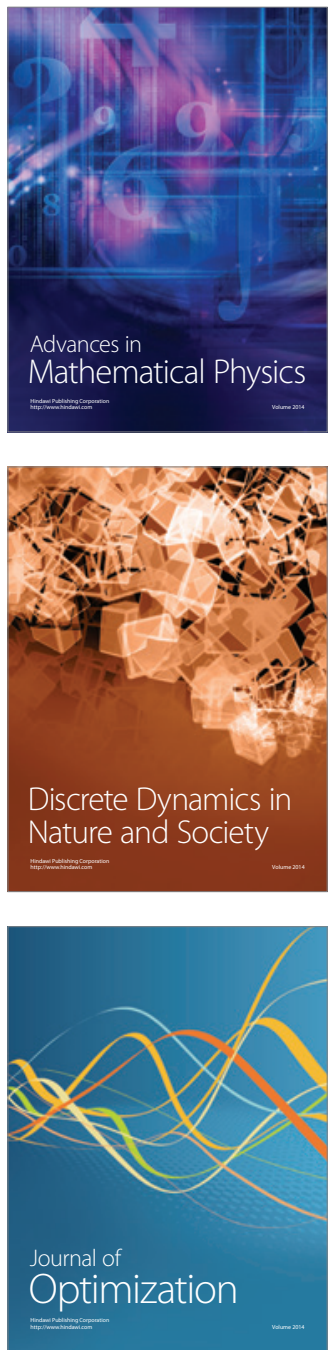